\title{
Publisher's Note: Topological spin-hedgehog crystals of a chiral magnet as engineered with magnetic anisotropy [Phys. Rev. B 96, 220414(R) (2017)]
}

N. Kanazawa, J. S. White, H. M. Rønnow, C. D. Dewhurst, D. Morikawa, K. Shibata, T. Arima, F. Kagawa, A. Tsukazaki, Y. Kozuka, M. Ichikawa, M. Kawasaki, and Y. Tokura

(Received 22 February 2019; published 28 February 2019)

DOI: 10.1103/PhysRevB.99.089902

This paper was published online on 28 December 2017 with the omission of a grant number in the acknowledgments section and an error in the acronym for the Swiss National Science Foundation. The acknowledgments on page 5 should read as follows:

We thank Y. Fujishiro and T. Yokouchi for their fruitful discussions. This work was supported by JSPS KAKENHI (Grants No. 24224009 and No. 15H05456), JST CREST (Grant No. JPMJCR16F1), the Swiss National Science Foundation (SNSF) Sinergia network "NanoSkyrmionics" (Grant No. CRSII5-171003), the SNSF Projects No. 153451 and No. 166298, and the European Research Council Project CONQUEST. We acknowledge the allocation of neutron beamtime at the Institut Laue Langevin (ILL), Grenoble, France and the Swiss Spallation Neutron Source (SINQ), Paul Scherrer Institute (PSI), Villigen, Switzerland.

The acknowledgments have been corrected as of 13 February 2019. The acknowledgments are incorrect in the printed version of the journal. 